\title{
O ESTADO DA ARTE DA SÍNTESE DE SEMICONDUTORES NANOCRISTALINOS COLOIDAIS
}

Fernanda Oliveira Silva, Lívia Cristina de Souza Viol, Diego Lourençoni Ferreira, José Luiz Aarestrup Alves e Marco Antônio Schiavon*

Departamento de Ciências Naturais, Universidade Federal de São João del-Rei, Campus Dom Bosco, Praça Dom Helvécio, 74, 36301-160 São João del-Rei - MG, Brasil

Recebido em 8/11/09; aceito em 21/4/10; publicado na web em 24/8/10

THE STATE OF THE ART IN THE SYNTHESIS OF COLLOIDAL SEMICONDUCTOR NANOCRYSTALS. Colloidal semiconductor nanocrystals, also known as quantum dots, have attracted great attention since they have interesting size-dependent properties due to the quantum confinement effect. These nanoparticles are highly luminescent and have potential applications in different technological areas, including biological labeling, light-emitting diodes and photovoltaic devices. The synthetic methods of semiconductor nanocrystals have progressed in the last 30 years, and several protocols were developed to synthesize monodisperse nanocrystals with good optical properties, different compositions and morphologies. This review describes the main methods used to synthesize nanocrystals in the II-VI and III-V systems, and the recent approaches in this field of research.

Keywords: semiconductor nanocrystals; quantum dots; colloidal synthesis.

\section{INTRODUÇÃO}

A síntese de nanocristais, partículas cristalinas com tamanhos de 1 a $100 \mathrm{~nm}$, tem sido o objeto de inúmeros trabalhos científicos no mundo todo, devido às suas intrigantes propriedades ópticas. Dentre os materiais nanométricos, têm-se os semicondutores nanocristalinos coloidais, também chamados de pontos quânticos, os quais apresentam os portadores de carga (buracos e elétrons) em um estado de forte confinamento quântico, o que faz com que estes materiais possuam propriedades ópticas que podem ser alteradas modificando-se apenas o tamanho das nanopartículas. Uma das propriedades ópticas mais interessantes destes nanocristais, do ponto de vista da aplicação tecnológica, é a forte intensidade de luminescência que apresentam, permitindo que sejam utilizados em dispositivos optoeletrônicos, fotovoltaicos e biomédicos, dentre outros. ${ }^{1,2}$

A Figura 1 apresenta imagens de dispersões coloidais aquosas de nanocristais de telureto de cádmio (CdTe) com diferentes cores de emissão, em função do tamanho das nanopartículas, bem como os espectros de absorção óptica (UV-Vis) e de fotoluminescência (PL) dessas amostras. ${ }^{3} \mathrm{Na}$ Figura $1 \mathrm{~b}$ é possível observar um deslocamento da banda de absorção óptica fundamental de 480 para $556 \mathrm{~nm}$ (red shift), devido à formação de partículas maiores ao longo da evolução da síntese. De maneira semelhante, na Figura 1c, fica evidente um deslocamento das bandas de emissão em direção a comprimentos de ondas maiores, de $507 \mathrm{~nm}$ da primeira amostra para $592 \mathrm{~nm}$ na última amostra, o que corresponde a uma variação nas cores de emissão, como mostrado na Figura 1a.

Os semicondutores nanocristalinos podem ser divididos em diferentes grupos da tabela periódica, tais como II-VI, III-V e IV-VI, sendo que, nesta revisão, são abordados, principalmente, os métodos experimentais de síntese para produção dos semicondutores dos grupos II-VI e III-V, embora esse último grupo seja ainda muito menos estudado. As sínteses destes materiais podem ser realizadas pelo método top-down, que utiliza técnicas físicas em que os nanocristais podem ser crescidos por litografia, ou pelo método bottom-up, que emprega técnicas de química coloidal. ${ }^{1,4}$ Por meio de técnicas físicas,

*e-mail: schiavon@ufsj.edu.br
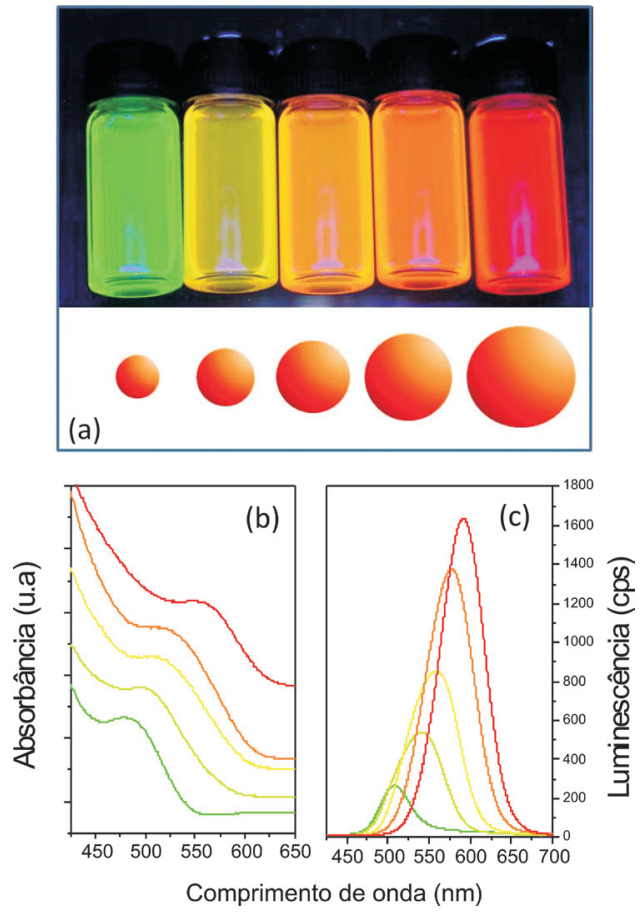

Figura 1. Variação das cores de emissão de dispersões coloidais aquosas de pontos quânticos de CdTe (a) e os correspondentes espectros de absorção óptica UV-Vis (b) e de fotoluminescência (c) dessas amostras

é possível produzir uma grande quantidade de material, no entanto, a obtenção de nanocristais com tamanhos uniformes, isto é, com uma distribuição estreita de tamanhos, torna-se comprometida quando se utilizam tais técnicas. Em contrapartida, por meio da química coloidal, é possível obter nanocristais com tamanhos relativamente uniformes, apesar de ser possível produzir apenas pequenas quantidades do material.

O principal objetivo deste artigo de revisão é apresentar os avanços ocorridos, nas últimas décadas, nos processos de síntese de semicondutores nanocristalinos II-VI e III-V, por meio dos métodos de química coloidal. 


\section{HISTÓRICO DO DESENVOLVIMENTO DAS SÍNTESES DE PONTOS QUÂNTICOS COLOIDAIS NO SISTEMA II-VI}

Os pontos quânticos têm sido obtidos, a partir da química coloidal, por meio de uma variedade de técnicas experimentais. Diversas rotas sintéticas têm sido propostas com o objetivo de aperfeiçoar e simplificar os métodos de síntese já existentes e, assim, tornar possível a obtenção de nanocristais com uma distribuição estreita de tamanhos, alta cristalinidade e sem defeitos de superfície. ${ }^{4}$

O primeiro método de síntese para obtenção de pontos quânticos, no sistema II-VI de CdTe e CdSe (seleneto de cádmio), surgiu na década de 80 , sendo baseado na imersão de certa quantidade de precursores metálicos e calcogenetos em um material vítreo. ${ }^{5}$ No entanto, esse método produziu uma baixa densidade de nanocristais e, além disso, não possibilitou um controle efetivo no tamanho desses materiais. Posteriormente, o crescimento desses materiais foi realizado em matrizes sintetizadas pelo processo sol-gel. Neste caso, foram utilizados poros umedecidos com géis dentro dos quais reagentes podiam ser difundidos e precipitados, sendo que o tamanho dos poros controlava tanto o tamanho máximo como a distribuição dos nanocristais. ${ }^{6}$

Mais tarde, a técnica de co-sputtering foi utilizada para a produção de pontos quânticos de $\mathrm{CdSe}$ e $\mathrm{Cd}_{\mathrm{x}} \mathrm{Mn}_{1-\mathrm{x}} \mathrm{Se}$. O crescimento foi realizado por meio de bombardeamento por radiofrequência (sputtering) de um alvo composto pelo material que constituiria o ponto quântico e pela matriz escolhida para confiná-lo. ${ }^{7} \mathrm{Em} 1996$, micelas começaram a ser utilizadas como moldes ou nanorreatores para controlar o tamanho dos nanocristais. Nanopartículas de sulfeto e seleneto de cádmio ( $\mathrm{CdS}$ e $\mathrm{CdSe}$ ) foram os primeiros materiais a terem seus tamanhos controlados pelo uso de micelas. ${ }^{8}{ }^{8}$ Por meio desta técnica, Quinlan et al. ${ }^{10}$ conseguiram obter nanopartículas de calcogenetos de cádmio com tamanhos entre 3,4 e 4,0 nm, exibindo boas propriedades de emissão. Apesar de ser ainda bastante utilizado, este método de síntese produz nanopartículas com baixa cristalinidade, uma vez que as reações não são realizadas em altas temperaturas, gerando baixos valores de rendimentos quânticos de luminescência. ${ }^{11}$

Um significativo progresso na síntese de pontos quânticos foi realizado na década de 90 , com o desenvolvimento de um novo método químico de síntese, proposto por Murray et al. ${ }^{12}$ que conseguiram obter nanocristais de CdS, CdSe e CdTe em uma faixa de tamanhos de 2 a 12 nm. Este método de síntese é baseado na rápida injeção de precursores organometálicos, como o dimetilcádmio, $\mathrm{Cd}\left(\mathrm{CH}_{3}\right)_{2}$, em um solvente coordenante, como óxido de trioctilfosfina (TOPO), a temperaturas elevadas. Neste caso, a nucleação das nanopartículas é a primeira etapa da síntese, sendo seguida pela etapa de crescimento, em temperaturas mais baixas, quando as concentrações dos monômeros decrescem. ${ }^{13}$ A Figura 2a ilustra a ocorrência dessas etapas no método de síntese organometálico, ressaltando as etapas de nucleação e crescimento das nanopartículas.

Além de ser possível obter amostras com uma distribuição estreita de tamanhos (dispersão entre 5 e 10\%), por meio do método organometálico, é possível também produzir nanopartículas cristalinas com rendimentos quânticos de fluorescência $(\phi f)$ de até $80 \%$. No entanto, este método de síntese conhecido como rota organometálica, ou método TOP/TOPO (trioctilfosfina/óxido de trioctilfosfina), utiliza precursores extremamente tóxicos, pirofóricos, de custo elevado e que necessitam de altas temperaturas de reação. ${ }^{12}$ Além disso, os nanocristais obtidos por essa rota sintética são praticamente insolúveis em meio aquoso e, portanto, não são compatíveis com o sistema biológico, o que é um fator limitante para a aplicação desses materiais em dispositivos biomédicos. Por esse motivo, pesquisas atuais têm proposto a modificação de superfície dos pontos quânticos para que os mesmos se tornem solúveis em meio aquoso. Entretanto, essa modificação é geralmente acompanhada por um decréscimo do rendimento quântico de fotoluminescência dos nanocristais. ${ }^{14}$

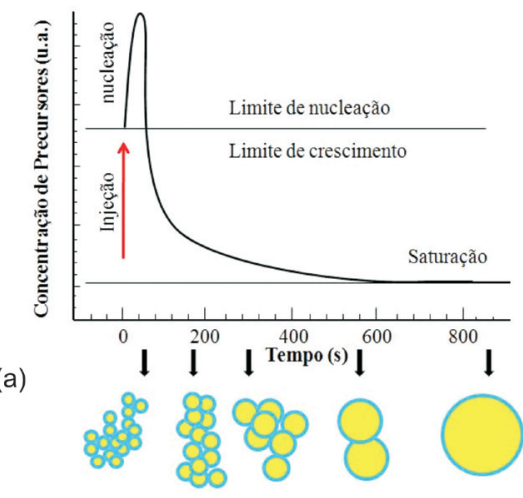

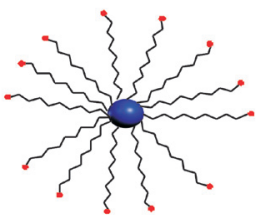

(b)

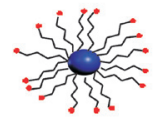

(c)
Figura 2. Principais etapas envolvidas nas sínteses de semicondutores nanocristalinos pelo método de injeção a quente desenvolvido por Murray et al. (a). ${ }^{12}$ Nanocristais estabilizados com ligantes de cadeia longa, solúveis em meio orgânico (b) e nanocristais estabilizados com ligantes de cadeia curta, solúveis em meio aquoso (c)

Diversos trabalhos foram realizados com o objetivo de tornar o procedimento experimental da síntese organometálica mais simples e eficiente. Peng e colaboradores ${ }^{14,15}$ propuseram a substituição do dimetilcádmio por óxido de cádmio, $\mathrm{CdO}$, ou por sais de cádmio contendo ânions de ácidos fracos, como o acetato de cádmio, $\mathrm{Cd}(\mathrm{Ac})_{2}$. Outra modificação adotada, na síntese de calcogenetos metálicos, foi a utilização da octadecilamina (ODA) como agente passivante e a substituição do solvente coordenante TOPO pelo solvente não coordenante 1-octadeceno (ODE). Este último apresenta menor custo, baixa toxicidade e, além disso, favorece a reatividade dos precursores de cádmio, apesar de também exigir altas temperaturas de reação. ${ }^{16}$ A ODA foi utilizada como ligante de superfície a fim de se diminuírem os defeitos de superfície dos nanocristais e evitar a agregação dos mesmos. Tais ligantes são comumente chamados de agentes passivadores ou surfactantes e são compostos por uma parte não polar (uma longa cadeia de hidrocarbonetos) e um grupo polar terminal (-SH, - $\mathrm{COOH},-\mathrm{NH})$, conforme ilustrado na Figura 2b. ${ }^{17,18}$

O comportamento desses ligantes, na superfície dos nanocristais, ainda não é totalmente compreendido e tem sido estudado com o intuito de controlar as propriedades ópticas desses materiais. Até o presente momento, sabe-se que os ligantes devem apresentar um comportamento dinâmico on/off em torno dos nanocristais, ora permitindo o crescimento desses nanomateriais (estado off ou desligados da superfície) e ora bloqueando o crescimento dos mesmos (estado on ou ligados à superfície). ${ }^{21}$ Recentemente, Peng e colaboradores ${ }^{17,18}$ estudaram o comportamento de alguns ligantes de superfície do tipo amina e comprovaram que o desempenho dinâmico dessas moléculas está intimamente relacionado à temperatura da reação, escolha adequada dos solventes, concentração e extensão da cadeia carbônica dos ligantes. Por exemplo, um aumento significativo na taxa de crescimento dos nanocristais foi observado quando se utilizaram temperaturas de reação acima das temperaturas de ebulição das aminas, uma vez que, neste caso, esses ligantes permaneceram mais tempo desligados da superfície e, portanto, favoreceram o crescimento dos mesmos.

Cao e Mulvaney propuseram a utilização de selênio elementar como precursor calcogeneto para a síntese de nanocristais de CdSe. 
Neste procedimento, o selênio em pó é dissolvido em ODE, a uma temperatura de $200^{\circ} \mathrm{C}$, sendo, posteriormente, adicionado a uma solução contendo o precursor de cádmio. Os nanocristais de CdSe obtidos desta forma apresentaram uma baixa distribuição de tamanhos (menor que 5\%) e uma estrutura cristalina do tipo blenda de zinco. ${ }^{19,20}$ Uma última simplificação foi proposta por Sapra et al. ${ }^{21}$ que utilizaram óleo de oliva como meio reacional para os precursores $\mathrm{CdO}$ e selênio elementar. A possibilidade de dissolver $\mathrm{CdO}$ em óleo de oliva foi, posteriormente, empregada para produção de nanocristais de CdSe, com tamanhos de 2,3 a 6,0 nm, utilizando ácido oleico (AO) como solvente da reação. ${ }^{21}$

Por meio de uma modificação do processo experimental, empregado por Murray et al. ${ }^{12}$ para obtenção de nanocristais de CdSe, Hines e Guyot-Sionnest conseguiram obter nanocristais de ZnSe. Neste caso, dietilzinco e selênio em pó foram dissolvidos em TOP e a hexadecilamina (HDA) foi utilizada como solvente da reação. A combinação TOP e HDA foi eficiente para remoção de defeitos de superfície dos nanocristais, o que resultou na obtenção de amostras estáveis e com excelentes propriedades de emissão. ${ }^{22}$

Peng e colaboradore ${ }^{23}$ reportaram a síntese de nanocristais de ZnSe, a partir da adição de uma solução estoque de selênio, preparada a partir da dissolução de selênio em pó em tributilfosfina (TBP) e ODE, em uma solução de estearato de zinco. Neste sistema, a ODA foi utilizada como ligante de superfície e a mistura tetracosano (TCA) e ODE foi utilizada como solvente da reação, a fim de se aumentar a temperatura da reação, uma vez que altas temperaturas são necessárias para o crescimento das nanopartículas de $\mathrm{ZnSe}$. Os nanocristais obtidos por esse procedimento apresentaram $\phi f$ superiores a $50 \%$.

É válido ressaltar a importância da substituição do cádmio pelo zinco, uma vez que o primeiro apresenta toxicidade elevada, o que dificulta sua manipulação e aplicação segura in vivo. ${ }^{2,24}$ Além disso, quando comparados a outros pontos quânticos como o CdS e CdSe, o ZnSe apresenta a vantagem de poder emitir na região do azul do espectro, o que o torna um material potencial para aplicação em biomarcadores. ${ }^{24}$

De modo geral, os métodos de síntese baseados em reagentes organometálicos têm possibilitado a obtenção de semicondutores nanocristalinos coloidais de alta qualidade, isto é, com baixa dispersão de tamanhos das nanopartículas, altos rendimentos quânticos de luminescência e estabilidade química. É visível a evolução dessa rota sintética no sentido de redução da toxicidade dos precursores e dos procedimentos experimentais, mesmo quando temperaturas elevadas são utilizadas.

\section{SEMICONDUTORES NANOCRISTALINOS COLOIDAIS SOLÚVEIS EM ÁGUA}

A busca pela simplificação dos procedimentos experimentais representa um dos principais objetivos da área de síntese de semicondutores nanocristalinos. Nesse sentido, Rogach et al..$^{25}$ foram os primeiros a descreverem a síntese de pontos quânticos de CdTe em meio aquoso. A síntese é baseada na injeção do precursor calcogeneto em uma solução aquosa, contendo o precursor metálico, seguida por um período de refluxo na temperatura de ebulição da água. Nesse sistema, grupos polifosfatos, tióis ou aminas de cadeias curtas são utilizados como ligantes, a fim de diminuir os defeitos de superfície dos nanocristais, como ilustrado na Figura 2c.

Posteriormente, Talapin et al. ${ }^{26}$ propuseram um novo método de síntese em meio aquoso capaz de produzir nanocristais de CdTe, a partir do precursor NaHTe. Nesse caso, primeiramente, o gás $\mathrm{H}_{2} \mathrm{Te}$ foi gerado por meio de uma reação entre $\mathrm{H}_{2} \mathrm{SO}_{4}$ e $\mathrm{Al}_{2} \mathrm{Te}_{3}$, sendo, posteriormente, borbulhado em uma solução de concentração determinada de $\mathrm{NaOH}$. Outra alternativa adotada por este grupo foi a injeção direta do gás $\mathrm{H}_{2}$ Te na solução precursora de cádmio. Mais tarde, Zhang et al. ${ }^{27}$ conseguiram obter nanocristais de CdTe, a partir do precursor $\mathrm{NaHTe}$, produzido por meio da redução de telúrio em pó com $\mathrm{NaBH}_{4}$. Apesar de esta ser uma rota alternativa para produção de nanocristais de $\mathrm{CdTe}$, a injeção direta do gás $\mathrm{H}_{2}$ Te é um método mais fácil, controlável e reprodutível, por meio do qual é possível obter nanocristais de alta qualidade..$^{28}$

A obtenção de nanocristais de $\mathrm{ZnSe}$ em meio aquoso é realizada de maneira semelhante à síntese dos nanocristais de CdTe. Gaponik et al..$^{29}$ descreveram o processo experimental para produção de nanocristais de $\mathrm{ZnSe}$, a partir do borbulhamento do gás $\mathrm{H}_{2} \mathrm{Se}$ em uma solução precursora de zinco. Da mesma maneira, Rogach et al. ${ }^{30}$ conseguiram obter nanocristais de $\mathrm{HgTe}$ e $\mathrm{Cd}_{\mathrm{x}} \mathrm{Hg}_{1-\mathrm{x}} \mathrm{Te}$ a partir da reação entre os íons $\mathrm{Hg}^{2+}$ e o gás $\mathrm{H}_{2} \mathrm{Te}$. As nanopartículas obtidas apresentaram uma ampla faixa de emissão espectral, de 700 a 1900 $\mathrm{nm}$. Recentemente, o grupo constituído pelos autores desta revisão relatou um estudo detalhado envolvendo a cinética de crescimento de nanocristais de CdTe, sintetizados em meio aquoso. ${ }^{31}$ Neste estudo, o crescimento das nanopartículas de CdTe seguiu a mesma cinética descrita por Peng e colaboradores ${ }^{32}$ para pontos quânticos solúveis em meio orgânico, em que a taxa de crescimento, a distribuição de tamanhos, o raio crítico e as constantes de difusão foram calculadas baseando-se no modelo cinético proposto por Sugimoto. ${ }^{33}$

Os procedimentos de síntese em meio aquoso têm sido amplamente utilizados para produção de semicondutores nanocristalinos, pois esses métodos apresentam boa reprodutibilidade sintética, menor custo, baixa toxicidade e são capazes de formar produtos facilmente solubilizados em água e, portanto, biocompatíveis. Além disso, os nanocristais, inicialmente solúveis em meio aquoso, podem ser facilmente solubilizados em solventes orgânicos por meio da troca do ligante de cadeia curta (Figura 2c) por um ligante de cadeia longa (Figura $2 b$ ). Rogach et $a l .{ }^{34}$ reportaram um processo em que nanocristais de CdTe, sintetizados em meio aquoso, foram solubilizados em tolueno, após processos de agitação e aquecimento simultâneos, na presença de acetona, em que o ligante de cadeia curta ácido tioglicólico foi substituído pelo ligante 1-dodecanotiol. O procedimento realizado possibilitou uma transferência de fase efetiva, uma vez que 90\% das nanopartículas tornaram-se solúveis em meio orgânico, apresentando propriedades ópticas semelhantes aos nanocristais solúveis em meio aquoso. Vale ressaltar que esta compatibilidade dos nanocristais com solventes orgânicos é de fundamental importância para aplicações desses materiais em dispositivos optoeletrônicos.

Apesar de apresentar inúmeras vantagens, o método de síntese coloidal em meio aquoso apresenta o inconveniente de produzir nanocristais polidispersos, ou seja, com uma distribuição larga de tamanhos, e com valores de $\phi$ relativamente baixos $(\sim 38-67 \%) .35,36$ Por essa razão, têm-se estudado, recentemente, algumas alternativas para tornar essa distribuição de tamanhos mais estreita e aumentar a eficiência quântica desses materiais sintetizados em meio aquoso.

Entre outros fatores, uma distribuição estreita de tamanhos pode ser requerida quando se deseja obter nanocristais com cores de emissão homogêneas, o que é muito interessante do ponto de vista das aplicações tecnológicas. Por esse motivo, algumas técnicas de pós-preparo têm surgido com o objetivo de diminuir a polidispersão de tamanhos dos nanocristais obtidos pelo método aquoso. Uma das técnicas mais empregadas com esse propósito é a precipitação seletiva de tamanhos, a qual foi inicialmente utilizada para a separação de tamanhos dos nanocristais de CdS. ${ }^{37}$ Tal técnica apresenta baixo custo e simplicidade experimental, sendo baseada na diferença de solubilidade de partículas com diferentes tamanhos.

O processo da precipitação seletiva de tamanhos é iniciado pela concentração da solução final, contendo os nanocristais de interesse. Posteriormente, um não solvente é adicionado ao produto final até que 
a solução apresente um aspecto turvo. Nessa etapa, as nanopartículas com tamanhos maiores são atraídas por forças de van der Waals e, por isso, tendem a se agregar e precipitar, primeiramente, sendo, então, separadas por centrifugação. Em seguida, uma nova quantidade do não solvente é adicionada ao sobrenadante para isolamento de uma segunda fração de nanopartículas. Esse procedimento pode ser realizado várias vezes até que se obtenham diversas frações de nanopartículas com uma distribuição de tamanhos mais estreita. ${ }^{38}$

Outras estratégias também têm sido adotadas para se elevar os baixos valores de $\phi f$ encontrados para os nanocristais sintetizados pelo método aquoso. A dopagem dos nanocristais e a utilização de processos de pós-preparo, como o tratamento fotoquímico, são alguns exemplos relevantes. ${ }^{39}$ Uma outra técnica adotada para este fim é baseada no crescimento de uma camada externa composta por uma matriz inorgânica também constituída de semicondutores nanocristalinos sobre a superfície dos nanocristais, dando origem a estruturas do tipo caroço/casca (core/shell) ${ }^{40}$ Essas mesmas estratégias têm sido utilizadas para melhorar a qualidade dos nanocristais preparados em meio orgânico, sendo, na maioria dos casos, desenvolvidos primeiramente para esses sistemas, e, posteriormente, aplicados aos sistemas coloidais aquosos.

\section{SEMICONDUTORES NANOCRISTALINOS DO TIPO CAROÇO/CASCA}

Os semicondutores nanocristalinos do tipo caroço/casca são formados por uma combinação de dois semicondutores distintos, em que um deles constitui o caroço (core), que é recoberto por uma camada externa contendo outro tipo de semicondutor (shell). Nesses sistemas, além de se elevar o rendimento quântico de fotoluminescência, tem-se também um ganho de estabilidade dos nanocristais. Além disso, a escolha adequada dos materiais do caroço e da casca permite estender a emissão desses nanomateriais para uma ampla faixa de comprimentos de onda do espectro. ${ }^{41}$ A Figura 3 a apresenta um exemplo de estruturas caroço-casca de CdTe/CdS.

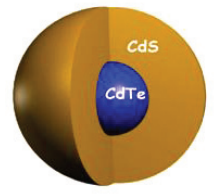

(a)

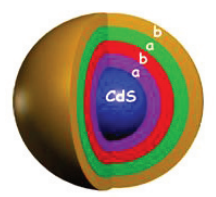

(c)

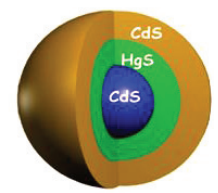

(b)

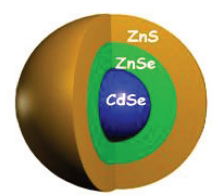

(d)
Figura 3. Esquema das estruturas do tipo caroço/casca de CdTe/CdS (a), poço quântico de pontos quânticos de CdS/HgS/CdS (b), duplas estruturas de pontos quânticos poços quânticos de $\mathrm{CdS} / \mathrm{HgS} / \mathrm{CdS} / \mathrm{HgS} / \mathrm{CdS}$ (c) em que " a" representa $\mathrm{HgS}$ e " $b$ " representa CdS, e estruturas caroço/casca/casca de CdSe/ZnSe/ZnS (d)

De acordo com o arranjo dos nanocristais e com a separação das bandas de energia, os sistemas caroço-casca podem ser classificados em tipo I ou tipo II, conforme esquematizado na Figura 4. No primeiro caso, tem-se na casca o semicondutor com maior band gap e, no caroço, o material com menor band gap (Figura 4a). Como resultado, buracos e elétrons ficam fortemente confinados na região do caroço, o que faz com que este tipo de material apresente rendimentos quânticos elevados, maior estabilidade e maior luminescência. Isso acontece pelo fato de o semicondutor da casca separar fisicamente a superfície ativa do semicondutor do caroço do meio reacional, o que provoca uma diminuição dos defeitos de superfície, que poderia comprometer a qualidade desses nanocristais.

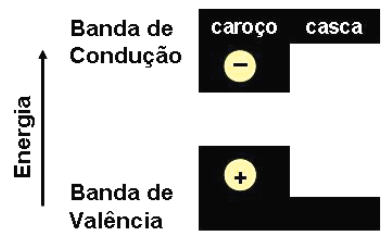

(a)

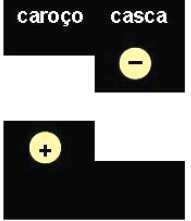

(b)
Figura 4. Representação esquemática das diferenças de energia entre as bandas de valência e bandas de condução e da posição dos elétrons e buracos nas diferentes estruturas caroço/casca do tipo I (a) e tipo II (b)

Hines e Guyot-Sionnest foram os primeiros a sintetizarem estruturas caroço/casca do tipo I baseadas em pontos quânticos de CdSe/ ZnS. ${ }^{41}$ Neste sistema, pontos quânticos de CdSe com tamanhos de $3 \mathrm{~nm}$ foram recobertos com uma e duas camadas de nanocristais de $\mathrm{ZnS}$, o que resultou em um aumento de $50 \%$ no $\phi f$ desses materiais quando comparado com as estruturas contendo apenas nanocristais de CdSe. Algumas modificações dessa síntese já foram realizadas por Jun e Jang, que conseguiram obter um aumento de $60 \%$ para esses sistemas caroço/casca. ${ }^{42}$ Peng e colaboradores ${ }^{43}$ reportaram a síntese de semicondutores de CdSe/CdS do tipo I, que apresentaram $\phi f$ superiores a $50 \%$ para nanocristais de CdSe com tamanhos de 2.3-3.9 nm. Pan et al. ${ }^{44}$ descreveram a síntese de pontos quânticos de $\mathrm{CdSe} / \mathrm{CdS}$ para nanocristais de CdSe com tamanhos de 1,2 a 1,5 nm e obtiveram $\phi f$ de até $80 \%$.

Os sistemas caroço-casca do tipo II são caracterizados por apresentarem uma separação espacial dos portadores de carga, uma vez que os elétrons ficam confinados na região da casca, e os buracos, na região do caroço, como ilustrado na Figura 4b. O principal interesse por sistemas do tipo II é a possibilidade de se alterarem as propriedades ópticas desses materiais pela variação da espessura da casca, o que ainda é pouco relatado para sistemas caroço-casca do tipo $\mathrm{I} \cdot{ }^{45} \mathrm{Os}$ sistemas caroço/casca do tipo II de $\mathrm{CdSe} / \mathrm{ZnSe}$ foram os primeiros a serem sintetizados, ${ }^{46}$ sendo que algumas modificações, realizadas por Reiss et al. ${ }^{47}$ proporcionaram um aumento de 60 para $85 \%$, no rendimento quântico de fotoluminescência, quando comparado com o CdSe. Mais tarde, Kim et al..$^{48}$ descreveram a síntese de sistemas caroço-casca do tipo II de CdTe/CdSe e CdSe/ZnTe. Para o sistema CdTe/CdSe, uma ampla faixa de comprimentos de onda de emissão (700-1100 nm) foi obtida com o aumento da espessura da casca e do tamanho do caroço.

Para que o processo de produção de sistemas caroço-casca seja realizado de maneira eficiente, alguns aspectos devem ser previamente estabelecidos. Primeiramente, a escolha dos materiais do caroço e da casca deve ser realizada de forma que a diferença de parâmetro de rede entre as duas estruturas seja pequena, a fim de se facilitar a cristalização desses materiais em uma mesma estrutura. Se essa condição não for adotada, o crescimento da casca poderá provocar a formação de defeitos de superfície, na interface caroço-casca, ocasionando a diminuição do $\phi f$ dos materiais resultantes. ${ }^{49} \mathrm{~A}$ Tabela 1 apresenta os valores de parâmetros de rede e $\mathrm{E}_{\text {gap }}$ para alguns nanocristais semicondutores. ${ }^{50}$

O controle da espessura da casca é um outro parâmetro essencial na produção de sistemas caroço/casca, sendo, portanto, de fundamental importância nos procedimentos experimentais. Se a espessura da 
Tabela 1. Parâmetros estruturais para alguns semicondutores nanocristalinos nos sistemas II-VI e III-V. Adaptada da ref. 46

\begin{tabular}{lccc}
\hline Material & Tipo & $\mathrm{E}_{\text {gap }}(\mathrm{eV})$ & Parâmetro de rede $(\AA)$ \\
\hline $\mathrm{ZnS}$ & II-VI & 3,61 & 5,41 \\
$\mathrm{ZnSe}$ & II-VI & 2,69 & 5,668 \\
$\mathrm{ZnTe}$ & II-VI & 2,39 & 6,104 \\
$\mathrm{CdS}$ & II-VI & 2,49 & $4,136 / 6,714$ \\
$\mathrm{CdSe}$ & II-VI & 1,74 & $4,3 / 7,01$ \\
$\mathrm{CdTe}$ & II-VI & 1,43 & 6,482 \\
$\mathrm{GaN}$ & III-V & 3,44 & $3,188 / 5,185$ \\
$\mathrm{GaP}$ & III-V & 2,27 & 5,45 \\
$\mathrm{GaAs}$ & III-V & 1,42 & 5,653 \\
$\mathrm{InN}$ & III-V & 0,8 & $3,545 / 5,703$ \\
$\mathrm{InP}$ & III-V & 1,35 & 5,869 \\
InAs & III-V & 0,35 & 6,058 \\
\hline
\end{tabular}

casca for fina, a passivação dos nanocristais do caroço será ineficiente, o que poderá resultar em uma instabilidade desses nanomateriais. Por outro lado, defeitos de superfície podem ser observados quando ocorre um aumento descontrolado na espessura do material da casca. ${ }^{51}$ Smith et al. ${ }^{45}$ relataram recentemente a conversão de CdTe/ZnSe do tipo I em tipo II, pelo aumento da espessura do material da casca. Nesse caso, este aumento da espessura da casca gerou uma tensão de compressão no material do caroço, transformando o sistema $\mathrm{CdTe} / \mathrm{ZnSe}$ em tipo II, o que foi confirmado experimentalmente pelo aumento do tempo de vida do estado excitado desta estrutura. Entretanto, tal comportamento precisa ainda ser mais bem avaliado para se conhecer o verdadeiro efeito da tensão de compressão da casca sobre o caroço na conversão de semicondutores do tipo I em tipo II.

Os sistemas caroço/casca podem ser sintetizados por meio de procedimentos experimentais que podem envolver uma ou duas etapas. Este último envolve, primeiramente, a síntese dos nanocristais do caroço, seguida por uma etapa de purificação e subsequente crescimento de 1-5 monocamadas do material da casca sobre o caroço. A purificação prévia do caroço é geralmente realizada por precipitação e os nanocristais obtidos são, posteriormente, dispersos em um solvente apropriado para crescimento da casca ao seu redor. A quantidade dos precursores necessária para uma espessura desejável da casca pode ser facilmente obtida pela determinação da concentração do caroço. Vale ressaltar que, nesse método de síntese, se tem a vantagem de se poder eliminar as impurezas dos produtos de reação, antes do processo de crescimento da casca, o que não acontece no procedimento realizado em uma única etapa de síntese (one-pot approach), em que nenhum processo de purificação é realizado e o crescimento da casca ocorre diretamente sobre o caroço recém-preparado. ${ }^{51}$

Peng e colaboradores ${ }^{52}$ propuseram o método SILAR (reação sucessiva de adsorção de camadas de íons) para o crescimento da casca de $\mathrm{CdS}$ ao redor do caroço de $\mathrm{CdSe}$. Tal método já era bastante utilizado para deposição de filmes finos em substratos sólidos e é baseado em sucessivas injeções de precursores catiônicos e aniônicos do material da casca sobre o caroço. Esse procedimento foi o adotado por Smith et al. ${ }^{45}$ para estudar a conversão de semicondutores do tipo I em tipo II, por meio do aumento da espessura da casca.

Algumas estruturas caroço/casca têm sido modificadas para obtenção de estruturas com multicamadas (multishell) a fim de se obterem propriedades ópticas ainda mais promissoras. Mews et al..$^{53,54}$ relataram a síntese de estruturas poço quântico de pontos quânticos (quantum dot quantum well) de $\mathrm{CdS} / \mathrm{HgS} / \mathrm{CdS}$ (Figura 3b) com propriedades ópticas fortemente dependentes das espessuras das múltiplas cascas de $\mathrm{HgS}$ e CdS. Em 2001, Braun et al. ${ }^{55}$ estenderam a síntese desses nanocristais e conseguiram obter estruturas duplas de pontos quânticos poços quânticos (double quantum dot quantum well) de CdS/HgS/CdS/HgS/CdS (Figura 3c).
Reiss et al. ${ }^{56}$ foram os primeiros a relatarem a síntese de estruturas caroço/casca/casca (core/shell/shell) de CdSe/ZnSe/ZnS (Figura 3d). Mais tarde, Talapin et al. ${ }^{57}$ obtiveram inicialmente nanocristais caroço/casca de CdSe/CdS e CdSe/ZnSe. Em ambos os casos, as estruturas foram recobertas por uma camada externa de $\mathrm{ZnS}$, havendo, portanto, a formação de CdSe/CdS/ZnS e CdSe/ $\mathrm{ZnSe} / \mathrm{ZnS}$. De acordo com os autores, a casca de $\mathrm{ZnS}$ é utilizada como camada externa, a fim de impedir a penetração dos portadores de carga para a superfície dos nanocristais. Além disso, defeitos de superfície poderiam ser formados entre o CdSe e $\mathrm{ZnS}$, devido à grande diferença de parâmetro de rede existente entre esses nanocristais $(\sim 1,87)$. Este problema é facilmente amenizado quando se coloca uma camada intermediária de $\mathrm{CdS}$ ou ZnSe entre o CdSe/ $\mathrm{ZnS}$, que atua diminuindo os defeitos de superfície e de tensão na interface desses materiais. Por esse motivo, estruturas do tipo caroço/casca apresentam elevados valores de rendimentos quânticos de fluorescência e alta estabilidade oxidativa, sendo, portanto, bastante estudados para fins biomédicos.

\section{SEMICONDUTOES NANOCRISTALINOS DOPADOS COM METAIS DE TRANSIÇÃO}

A dopagem de semicondutores nanocristalinos corresponde à introdução de uma pequena quantidade de "impurezas" dentro da rede cristalina do material e é outra estratégia que tem sido investigada visando a otimização das propriedades ópticas dos semicondutores nanocristalinos. Um exemplo, comumente relatado na literatura, é a dopagem de semicondutores II-VI com íons paramagnéticos como o $\mathrm{Mn}^{2+}(\mathrm{S}=5 / 2)$, o que faz com que esses materiais sejam estáveis e também apresentem interessantes propriedades magnéticas. ${ }^{58}$ Nanocristais de $\mathrm{ZnSe}$ dopados com manganês (ZnSe:Mn), por exemplo, são termicamente estáveis e apresentam $\phi f$ de até $70 \%$. A Figura 5 apresenta os espectros de absorção e emissão para o ZnSe e ZnSe:Mn, em que é possível observar que o método de dopagem é eficiente, produzindo $100 \%$ de nanocristais dopados, sem sinais de emissão do ZnSe. Além disso, é possível verificar que o sistema $\mathrm{ZnSe:Mn}$ apresenta uma grande separação entre a absorção e emissão, o que o torna bastante interessante do ponto de vista tecnológico.
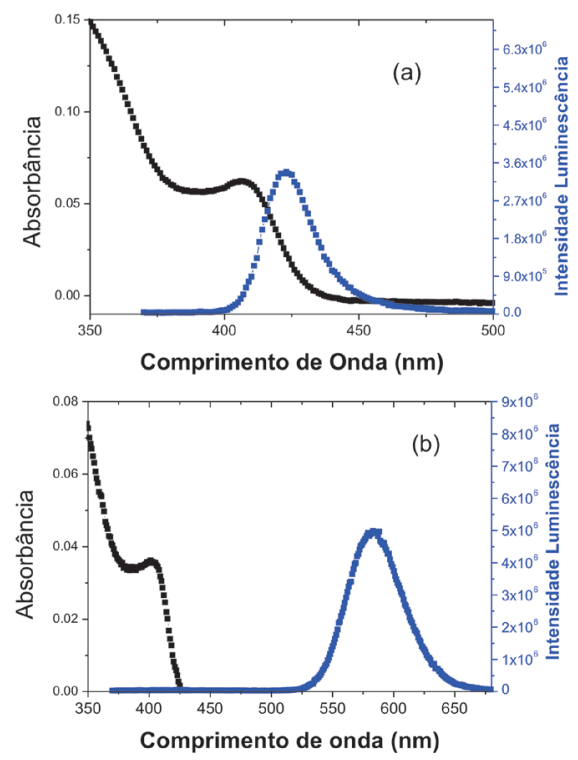

Figura 5. Espectros de absorção e emissão para pontos quânticos de ZnSe (a) e ZnSe:Mn (b) 
Bhargava et al. ${ }^{59}$ foram os primeiros a relatarem a síntese de nanopartículas de $\mathrm{ZnS}: \mathrm{Mn}$, que apresentaram excelentes propriedades de emissão, mesmo quando os nanocristais de $\mathrm{ZnS}$ não eram de alta qualidade. Peng e colaboradores ${ }^{60}$ descreveram algumas rotas sintéticas para a produção de $\mathrm{ZnSe}$ dopados com $\mathrm{Cu}$ e $\mathrm{Mn}$, que apresentaram um aumento no rendimento quântico de fotoluminescência de até $70 \%$, quando comparados com os sistemas sem o dopante.

No processo de dopagem, é de fundamental importância que os íons adicionados apresentem a mesma valência e, além disso, possuam raios iônicos semelhantes aos íons hospedeiros, a fim de se evitar a migração dos dopantes para a superfície, muitas vezes realizada pelos próprios hospedeiros como um processo natural de purificação. ${ }^{61}$ Recentemente, Erwin et al. ${ }^{62}$ relataram um estudo cinético para o entendimento do processo de dopagem em nanocristais semicondutores. O modelo propõe que somente as impurezas remanescentes, na superfície dos nanocristais, por um período de tempo comparável à taxa de crescimento dos mesmos, devem ser incorporadas dentro desses materiais. Vale ressaltar que a morfologia da superfície, a forma dos nanocristais e a natureza dos ligantes são alguns fatores que influenciam o tempo de residência das impurezas na superfície dos nanocristais e, portanto, são de fundamental relevância para o processo de dopagem. ${ }^{60}$

Murphy et al. ${ }^{63}$ relataram um estudo envolvendo as mudanças das propriedades ópticas de nanopartículas de $\mathrm{ZnS}$, quando as mesmas foram submetidas à dopagem com íons $\mathrm{Mn}^{2+}$ "pelo lado de fora" $\mathrm{e}$ "pelo lado de dentro" da rede cristalina desse material. O esquema ilustrativo apresentado na Figura 6 ilustra esses diferentes tipos de dopagem. Os autores verificaram que a localização dos íons $\mathrm{Mn}^{2+} \mathrm{de}-$ termina as propriedades ópticas do material hospedeiro, uma vez que as nanopartículas de $\mathrm{ZnS}$ apresentaram emissões nas regiões laranja e ultravioleta do espectro, quando a adição do íon foi realizada "pelo lado de fora" e "pelo lado de dentro" da rede cristalina, respectivamente. Além disso, tempos de vida de decaimento de fotoluminescência mais curtos foram obtidos quando a dopagem foi realizada "pelo lado de fora" dos nanocristais. Isso ocorre devido a uma reorganização dos estados emissores, que é verificada apenas quando os íons $\mathrm{Mn}^{2+}$ estão localizados na superfície do material hospedeiro.

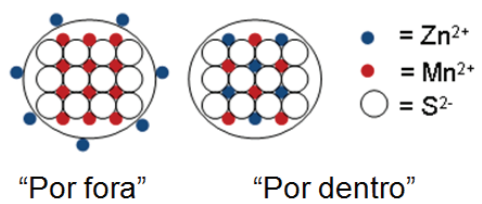

Figura 6. Esquema ilustrativo das duas maneiras distintas de se realizar a dopagem com íons $\mathrm{Mn}^{2+}$ em nanocristais de $\mathrm{ZnS}$

\section{NANOCRISTAIS SEMICONDUTORES NO SISTEMA III-V}

Apesar de os elementos do grupo III apresentarem propriedades eletrônicas mais promissoras e baixa toxicidade, quando comparados aos elementos do grupo II, os processos experimentais empregados na síntese dos semicondutores III-V são mais complexos do que os utilizados para produção dos semicondutores II-VI, uma vez que os primeiros exigem temperaturas muito elevadas e tempos de reação prolongados. ${ }^{64}$

A maioria dos processos de produção dos nanocristais semicondutores III-V surgiu como uma adaptação dos métodos já existentes para síntese de nanocristais II-VI. Nozik et al. ${ }^{65}$ relataram a preparação de nanopartículas de InP, a partir de cloroíndio e $\mathrm{P}\left[\mathrm{Si}\left(\mathrm{CH}_{3}\right)_{3}\right]_{3} \mathrm{em}$ uma mistura de TOPO e TOP a $270{ }^{\circ} \mathrm{C}$. Os nanocristais sintetizados apresentaram um baixo valor de rendimento quântico de fotoluminescência $(\sim 1 \%)$ apesar de a distribuição de tamanhos ser um pouco estreita ( 10\%), o que é causado por defeitos de superfície. Mais tarde, Peng e colaboradores ${ }^{64}$ propuseram uma modificação desse método e conseguiram elevar o $\phi f$ desses nanocristais, por meio da utilização de solventes não coordenantes como o 1-octadeceno.

Micic et al. ${ }^{66}$ foram os primeiros a relatarem a síntese de nanocristais monodispersos de $\mathrm{GaP}$, produzidos, a partir de $\mathrm{Ga}\left(\mathrm{P} t \mathrm{Bu}_{2}\right)_{3}$, em uma mistura de trioctilamina (TOA) e hexadecilamina (HDA). Alguns semicondutores do tipo III-V apresentam processos de preparação complicados devido a pouca disponibilidade dos precursores necessários. Nanocristais de nitrito (AlN, InN, GaN), por exemplo, são de difícil obtenção por exigirem altas temperaturas de crescimento e baixas temperaturas de decomposição. Recentemente, Rao et al. relataram a síntese de nanocristais de AlN e InN com uma distribuição estreita de tamanhos a partir da decomposição térmica de um complexo metal-ureia, em refluxo com trioctilamina, sob atmosfera de nitrogênio. ${ }^{67}$

\section{CONSIDERAÇÕES FINAIS}

Os processos de preparação dos nanocristais semicondutores têm sido constantemente modificados, a fim de se obterem procedimentos experimentais cada vez mais simples e capazes de produzirem nanopartículas com excelentes propriedades ópticas. A síntese em meio aquoso, por exemplo, surgiu como uma simplificação do método de síntese em meio orgânico, sendo necessária a utilização de algumas estratégias para que a qualidade dos nanocristais fosse mantida. Dessa forma, nos dias atuais, é possível produzir nanopartículas monodispersas, cristalinas e altamente luminescentes, por meio de técnicas experimentais menos tóxicas, de menor custo e com boa reprodutibilidade sintética. O maior desafio dessa área de pesquisa talvez seja a obtenção de um processo experimental economicamente viável, capaz de produzir nanocristais semicondutores, nos sistemas II-VI e III-V, em larga escala para que, finalmente, os mesmos possam ser aplicados industrialmente em dispositivos optoeletrônicos, fotovoltaicos e biomédicos.

\section{AGRADECIMENTOS}

À CAPES, CNPq, FAPEMIG, Instituto de Nanotecnologia (MCT/ $\mathrm{CNPq})$ e à Fulbright Brasil.

\section{REFERÊNCIAS}

1. Park, J.; Joo, J.; Kwon, S. G.; Jang, Y.; Hyeon, T.; Angew. Chem., Int. Ed. 2007, 46, 4630.

2. Rogach, A. L.; Semiconductor Nanocrystal Quantum Dots: Synthesis, Assembly, Spectroscopy and Application, Springer Wien: New York, 2008, cap. 1.

3. Ferreira, D. L.; Dissertação de Mestrado, Universidade Federal de São João Del Rei, Brasil, 2007.

4. Rogach, A. L.; Talapin, D. V.; Schevchenko, E. V.; Kornowski, A.; Haase, M.; Weller, H.; Adv. Funct. Mater. 2002, 10, 653.

5. Ekimov, A. I.; Onuschenko, A. A.; Sov. Phys. Semicond. 1982, 16, 1215.

6. Arai, T.; Fijurmira, H.; Umezu, I.; Ogawa, T.; Fuji, A.; J. Appl. Phys. 1989, 28, 484.

7. Daudin, B.; Widmann, F.; Fenillet, G.; Sanson, Y.; Pelekanos, N.; Mater. Sci. Eng., B 1999, 59, 330.

8. Pileni, M. P.; Langmuir 1997, 13, 3266.

9. Ge, J. P.; Chen, W.; Liu, L.; Chem. Eur. 2006, 12, 6552.

10. Quinlan, F. T.; Kuther, J.; Tremel, W.; Knoll, W.; Risbud, S.; Stroeve, P.; Langmuir 2000, 16, 4049.

11. Pileni, M. P.; Adv. Funct. Mater. 2001, 11, 136.

12. Murray, C. B.; Norris, D. J.; Bawendi, M. G.; J. Am. Chem. Soc. 1993, $115,8706$. 
13. Donegá, M. C.; Liljeroth, P.; Vanmaekelbergh, D.; Small 2005, 1, 1152.

14. Peng, Z. A.; Peng, X.; J. Am. Chem. Soc. 2001, 123, 183.

15. Qu, L.; Peng, Z. A.; Peng, X.; Nano Lett. 2001, 1, 333.

16. Yu, W.; Peng, X.; Angew. Chem., Int. Ed. 2002, 41, 2368.

17. Xiaohui, J.; Copenhaver, D.; Peng, X.; J. Am. Chem. Soc. 2008, 130, 5726.

18. Pradhan, N.; Reifsnyder, D.; Xie, R.; Aldana, J.; Peng, X.; J. Am. Chem. Soc. 2007, 129, 9500.

19. Yang, Y. A.; Williams, K. R.; Cao, Y. C.; Angew. Chem., Int. Ed. 2005, $44,6712$.

20. Jasieniak, J.; Bullen, C.; Embden, J.; Mulvaney, P.; J. Phys. Chem. B 2005, 109, 20665.

21. Sapra, S.; Rogach, A. L.; Feldmann, J.; J. Mater. Chem. 2006, 16, 3391.

22. Hines, M. A.; Guyot-Sionnest, P.; J. Phys. Chem. B 1998, 102, 3655.

23. Peng, X.; Pradhan, N.; Wang, Y.; Li, S, L.; Nano Lett. 2004, 4, 2262.

24. Andrade, J. J.; Brasil, A. G.; Farias, P. M. A.; Fontes, A.; Santos, B. S.; Microeletronics Journal 2009, 40, 641.

25. Rogach, A. L.; Katsikas, L.; Kornowski, A.; Su, D.; Eychmuller, A.; Weller, H.; Ber. Bunsenges. Phys. Chem. 1996, 100, 1772.

26. Gaponik, N.; Talapin, D. V.; Rogach, A. L.; Hoppe, K.; Weller, H.; J. Phys. Chem. B 2002, 106, 7177.

27. Zhang, H.; Wang, L.; Xiong, H.; Hu, L.; Yang, B.; Li, W.; Adv. Funct. Mater. 2003, 15, 1712.

28. Zhang, H.; Zhou, Z.; Yang, B.; Gao, M.; J. Phys. Chem. B 2003, 107, 8.

29. Shavel, A.; Gaponik, N.; J. Phys. Chem. B 2004, 108, 5905.

30. Rogach, A. L.; Eychmuller, A.; Hickey, S. G.; Kershaw S. V.; Small 2007, 3, 536.

31. Ferreira, D. L.; Silva, F. O.; Viol, L. C. S.; Licínio, P.; Valadares, M.; Cury, L. A.; Schiavon, M. A.; Alves, J. L. A.; J. Chem. Phys. 2009, 131, 084712 .

32. Peng, X.; Wickham, J.; Alivisatos, A. P.; J. Am. Chem. Soc. 1998, 120, 5343.

33. Sugimoto, T.; Adv. Colloid Interface Sci. 1987, 28, 65.

34. Rogach, A. L.; Gaponik, N.; Talapin, D. V.; Weller, H.; Nano Lett. 2002, 2, 803.

35. Li, L.; Qian, H.; Fang, N.; Ren, J.; J. Lumin. 2006, 116, 59.

36. Zhang, H.; Cui, Z.; Wang, Y.; Zhang, K.; Ji, X.; Lu, C.; Yang, B.; Gao, M. Y.; Adv. Funct. Mater. 2003, 15, 777.

37. Chemseddine, A.; Weller, H.; Phys. Chem. 1993, 97, 636.

38. Rogach, A. L.; Semiconductor Nanocrystal Quantum Dots: Synthesis, Assembly, Spectroscopy and Application, Springer Wien: New York, 2008, cap. 3 .

39. Li, C. L.; Nishikawa, K.; Ando, M.; Enomoto, H.; Murase, N.; Colloids Surf., A 2007, 294, 22.

40. Talapin, D. V.; Poznyak, S. K.; Gaponik, N. P.; Physica E 2002, 14, 237.

41. Hines, M. A.; Guyot- Sionnest, P.; J. Phys. Chem. 1996, 100, 468.
42. Jun, S.; Jang, E.; Chem. Commun. 2005, 36, 4616.

43. Peng, X. G.; Schlamp, M. C.; Kadavanich, A. V.; Alivisatos, A. P.; J. Am. Chem. Soc. 1997, 119, 7019.

44. Pan, D. C.; Wang, Q.; Jiang, S. C.; Ji, X. L.; An, L. J.; Chem. Mater. 2005, 17, 176.

45. Smith, A. M.; Mohs, A. M.; Nie, S.; Nanotechnology 2009, 4, 56.

46. Danek, M.; Jensen, K. F.; Murray, C. B.; Bawendi, M. G.; Chem. Mater. 1996, 8, 173.

47. Reiss, P.; Bleuse, J.; Pron, A.; Nano Lett. 2002, 2, 781.

48. Kim, S.; Fisher, B.; Eisler, H. J.; Bawendi, M.; J. Am. Chem. Soc. 2003, $125,11466$.

49. Chen, X. B.; Lou, Y. B.; Samia, A. C.; Burda, C.; Nano Lett. 2003, 3 , 799.

50. Madelung, O.; Schulz, M.; Weiss, H.; Landolt-Bornstein: numerical data and functional relationships in science and technology, new series, group III: crystals and solid state physics, Springer, 1982, vol. 3, (17b).

51. Rogach, A. L.; Semiconductor Nanocrystal Quantum Dots: Synthesis, Assembly, Spectroscopy and Application, Springer Wien: New York, 2008, cap. 2.

52. Li, J. J.; Wang, Y. A.; Guo, W. Z.; Keay, J. C.; Mishima, T.D.; Johnson, M. B.; Peng, X. G.; J. Am. Chem. Soc. 2003, 125, 12567.

53. Eychmuller, A.; Mews, A.; Weller, H.; Chem. Phys. Lett. 1993, 208, 59.

54. Mews, A.; Eychmuller, A.; Giersig, M.; Schooss, D.; Weller, H.; J. Phys. Chem. 1994, 98, 934.

55. Braun, M.; Burda, C.; El-Sayed, M. A.; J. Phys. Chem. A 2001, 105, 5548.

56. Reiss, P.; Carayon, S.; Bleuse, J.; Pron, A.; Synth. Met. 2003, 139, 649.

57. Talapin, D. V.; Mekis, I.; Gotzinger, S.; Kornowski, A.; Benson, O.; Weller, H.; J. Phys. Chem. B 2004, 108, 18826.

58. Furdyna, J. K.; J. Appl. Phys. 1988, 64, 29.

59. Bhargava, R. N.; Gallagher, D.; Hong, X.; Nurmikko, A.; Phys. Rev. Lett. 1994, 72, 416.

60. Pradhan, N.; Battaglia, D. M.; Lui, Y.; Peng, X.; Nano Lett. 2007, 7, 312.

61. Reiss, P.; New J. Chem. 2007, 31, 1843.

62. Erwin, S. C.; Zu, L.; Haftel, M. I.; Efros, A. L.; Kennedy, T. A.; Norris, D. J.; Nature 2005, 436, 91 .

63. Sooklal, K.; Cullum, B. S.; Angel, S. M.; Murphy, C. J.; J. Phys. Chem. 1996, $100,4551$.

64. Peng, X.; Battaglia, D.; Nano Lett. 2002, 2, 1027.

65. Nozik, A. J.; Micic, O. I.; Curtis, C. J.; Jones, L. M.; Sprague, J. R.; J. Phys. Chem. 1994, 98, 4996.

66. Micic, O. I.; Sprague, J. R.; Curtis, C. J.; Jones, K. M.; Machol, J. L.; Nozik, A. J.; J. Phys. Chem. 1995, 99, 7754.

67. Rao, C. N. R.; Sardar, K.; Dan, M.; Schwenzer, B.; J. Mater. Chem. 2005, 15, 2175. 